\title{
Inventaire Et Évaluation Scientifique Des Géosites Des Falaises De Fresco En Côte d'Ivoire
}

\author{
Wrohou Bénédicte Dibo \\ Ezzoura Errami \\ N'Goran Jean-Paul Yao
}

Département de Géosciences Marines, UFR des Sciences de la Terre et des Ressources Minières, Université Félix Houphouët Boigny, Abidjan, Côte d'Ivoir

Faculté Polydisciplinaire de Safi, Université Cadi Ayyad, Marrakech, Maroc

\section{Kouamé Léger Djeya \\ Juliette Tea \\ Gboméa Marcelle Gauly \\ Zéli Bruno Digbehi}

Département de Géosciences Marines, UFR des Sciences de la Terre et des Ressources Minières, Université Félix Houphouët Boigny, Abidjan, Côte d'Ivoire

Société Nationale d’Opération Pétrolière de Côte d’Ivoire

\section{Doi:10.19044/esj.2021.v17n25p150}

Submitted: 20 March 2021

Accepted: 21 June 2021

Published: 31 July 2021
Copyright 2021 Author(s)

Under Creative Commons BY-NC-ND 4.0 OPEN ACCESS

Cite As:

Dibo.W.B., Errami E., Jean-Paul Yao N’G., Djeya K.L., Tea J., Gauly G.M. \& Digbehi Z.B(2021). Inventaire Et Évaluation Scientifique Des Géosites Des Falaises De Fresco En Côte d'Ivoire. European Scientific Journal, ESJ, 17(25), 150.

https://doi.org/10.19044/esj.2021.v17n25p150

\section{Résumé}

Plusieurs pays africains ont adhéré au Réseau Africain de Géoparc (AGN) et ont entrepris de nombreuses études sur le géopatrimoine suite aux investigations menées par cette organisation pour promouvoir les richesses naturelles des pays africains. Les falaises de Fresco, premiers sites identifiés en Côte d'Ivoire, sont connues pour leurs richesses géologiques et écologiques. Ainsi, une étude bibliographique approfondie et complétée par l'analyse des données géologiques sur l'ensemble de ces affleurements ont permis de faire l'inventaire des éléments de valeur des falaises et de les évaluer 
sur le plan scientifique. L'évaluation de ces géosites (ensemble des cinq falaises) a livré une valeur moyenne de $0,7 / 1$ et a ainsi confirmé leur importance en tant que géosites potentiels. L'environnement naturel aux alentours des falaises renferme une riche biodiversité de végétation littorale abritant de nombreuses espèces animales dont certaines sont en voie de disparition. Cette étude aura permis d'évaluer les falaises comme élément important de la géodiversité pouvant favoriser la promotion du géotourisme et contribuer au développement socio-économique de la région de Fresco.

Keywords: Géopatrimoine, Falaises, Valeur Scientifique, Biodiversité, Fresco, Géoparc, AGN, Géotourisme

\section{Inventory And Scientific Assessment Of The Geosites Of The Cliffs Of Fresco In Côte d'Ivoire}

\section{Wrohou Bénédicte Dibo \\ Ezzoura Errami \\ N'Goran Jean-Paul Yao}

Département de Géosciences Marines, UFR des Sciences de la Terre et des Ressources Minières, Université Félix Houphouët Boigny, Abidjan, Côte d'Ivoir

Faculté Polydisciplinaire de Safi, Université Cadi Ayyad, Marrakech, Maroc

\section{Kouamé Léger Djeya}

Juliette Tea

Gboméa Marcelle Gauly

Zéli Bruno Digbehi

Département de Géosciences Marines, UFR des Sciences de la Terre et des Ressources Minières, Université Félix Houphouët Boigny, Abidjan, Côte d'Ivoire

Société Nationale d’Opération Pétrolière de Côte d’Ivoire

\section{Abstract}

Several African countries have joined the African Geopark Network (AGN) and have undertaken numerous studies on geoheritage following the investigations carried out by this organization to promote the natural resources of African countries. The Fresco escarpments, the first site identified in Côte d'Ivoire, are known for their geologic and biologic wealth. Thus, an in-depth bibliographical study completed by the analysis of geological data made it possible to make an inventory of the elements of geodiversity of the five escarpments and to evaluate their scientific value. The evaluation of these geosites (all five cliffs) yielded an average of $0.7 / 1$; and confirmed their 
importance as potential geosites. The natural environment around the cliffs contains a rich biodiversity of coastal vegetation, home to many animal species that some of them are endangered. This study will have made it possible to assess the cliffs as an important element of geodiversity that can allow the promotion of geotourism and contribute to the socio-economic development of the Fresco region.

Keywords: Geoheritage, Cliffs, Scientific Value, Biodiversity, Fresco, Geopark, AGN, Geotourism

\section{Introduction}

L'inventaire, la valorisation, la conservation et la promotion des sites géologiques en Afrique qui ont des atouts exceptionnels (scientifiques, pédagogiques, culturels et esthétiques) à l'échelle locale, nationale et internationale, est un des objectifs principaux visés par le Réseau Africain de Géoparcs nommé en anglais “African Geoparks Network (AGN)”. Ce dernier a été créé par l'Association Africaine des Femmes en Géosciences (AAWG) lors de la préparation de sa $5^{\text {ème }}$ conférence tenue en 2009 à Abidjan (Côte d'Ivoire). Ainsi, plusieurs travaux portant sur le géopatrimoine et la création de géoparcs ont été entrepris dans différents pays africains (Maroc, Ethiopie, Algérie, Sénégal, Côte d’Ivoire, Cameroun, Tanzanie, Afrique du Sud, etc.). Aussi, l'AGN a organisé en association avec d'autres partenaires plusieurs tables rondes, conférences, groupes de travail et visites de sites dans différents pays africains (Errami et al., 2013). Le but ultime en est de sensibiliser les populations et les autorités locales et les gouvernements de chaque pays sur l'importance de leur géopatrimoine comme outil potentiel de développement socio-économique local durable à travers la promotion du géotourisme et la création de géoparcs. Ainsi, les éléments de la géodiversité (roches, minéraux, fossiles, reliefs...) pourront être utilisés d'une manière durable qui, tout en assurant leur conservation, leur permet de jouer pleinement leur rôle de développement humain et socio-économique local durable. Selon l'AGN, la création de géoparc pourrait être un facteur de développement socioéconomique local à travers la création de routes, l'électrification, l'apport en eau potable ; mais aussi l'exploitation des produits locaux et la promotion des richesses culturelles et la promotion d'une nouvelle niche touristique, le géotourisme. Les géoparcs constituent aussi une source de cohésion sociale et de paix dans la région, à l'échelle nationale ainsi qu'entre pays africains (Errami et al. 2015).

Toutes ces investigations de l'AGN ont favorisé l'élan considérable depuis quelques années des pays déjà visités à travers des études entreprises portant sur l'inventaire de leurs géosites potentiels. En effet, l'AGN a débuté ses activités un an après sa création en Côte d’Ivoire en 2010 ; et pendant la 
$5^{\text {ème }}$ conférence de l'AAWG, les falaises de Fresco ont été visitées et identifiées comme étant des géosites pouvant être valorisés à l'échelle locale, nationale, voire internationale. La Côte d'Ivoire a ratifié la convention pour la protection du patrimoine mondial culturel et naturel de l'UNESCO en 1981 et compte cinq biens patrimoniaux : le parc national de Taї (1982), la ville historique de Grand-Bassam (2012), la danse Zaouli en pays Gouro (2017, le plus récent), le parc national de la Comoé (1983), la réserve naturelle intégrale du mont Nimba (1981). Les deux derniers étant depuis peu sur la liste du patrimoine mondial en péril pour manque d'assistance conforme aux termes de la convention.

Le choix porté sur les falaises de Fresco est lié à leur importance scientifique. Du point de vue stratigraphique, elles représentent le seul site où affleurent les formations marines (argiles et calcaires glauconieux) d'âge Paléocène-Eocène dans le bassin sédimentaire sud-ouest de la Côte d'Ivoire (Martin \& Tastet, 1972). Ces falaises présentent un contenu fossilifère riche auquel sont associées de nombreuses figures sédimentaires (stratifications obliques, hardgrounds, bioturbations) ; elles ont fait l'objet de nombreux travaux géologiques (Remy, 1960 ; Tessier, 1960 ; Lys, 1961 ; Tastet \& Roques, 1969 ; Yao, 2012) et ayant fournis des informations importantes sur le plan sédimentologique, paléontologique et stratigraphique (lithostratigraphie du bassin sud-ouest, l'âge relatif des formations, etc.). Par ailleurs, les indices d'hydrocarbures découverts à travers les argiles noires bitumineuses au niveau de ces falaises ont suscité un intérêt et des campagnes d'exploration des sociétés pétrolières notamment la PETROCI. Le but de ce travail est d'identifier, d'inventorier et d'évaluer la géodiversité des falaises de Fresco dans le but de montrer leur richesse en tant qu'élément important du géopatrimoine. L'évaluation scientifique de ces géosites, complétée par un inventaire général de la biodiversité et des richesses culturelles et socioéconomiques de la zone permet d'accorder une attention particulière à la région pouvant jouer un rôle capital dans la création du géoparc sud-ouest ivoirien.

\section{Cadre Geographique \\ Localisation}

Fresco est une ville située au sud-ouest de la Côte d'Ivoire à environ 200 km de la ville d'Abidjan sur le littoral dans la région du Gbôklè. Elle est limitée à l'ouest par la ville de Sassandra, à l'est par la ville de Grand-Lahou, au Nord par la ville de Divo et au Sud en contact direct avec le golfe de Guinée. Fresco ( $\left.5^{\circ} 05^{\prime} 00^{\prime \prime} \mathrm{N} / 5^{\circ} 34^{\prime} 00^{\prime \prime} \mathrm{W}\right)$ s'étend sur une superficie de $3255 \mathrm{~km}^{2}$ et compte près de 41058 habitants (RGPH 2014). Le nom "Fresco" désigne une localité au Portugal, a été donné à cette localité en Côte d'Ivoire, vers 1472 par un explorateur portugais qui naviguait dans le golfe de Guinée 
pour des échanges commerciaux. En 1967, du fait des menaces constantes de la mer, les populations ont dû quitter les côtes (ancien site Fresco-village) pour s'installer sur la terre ferme. Aujourd'hui, la nouvelle ville de Fresco surplombe la lagune N'gni appelée aussi la lagune de Fresco.

\section{Géographie physique}

Fresco est situé sur le littoral ivoirien et est par conséquent soumis aux conditions naturelles et environnementales de ce milieu. Le littoral s'étend du cap des Points à l'Est vers le cap des Palmes à l'Ouest et est limité au Nord par la route côtière à l'ouest d'Abidjan et la route de Noé en passant par Alépé à l'est. Dans la partie sud, il est délimité par l'isobathe $120 \mathrm{~m}$. Sa superficie est estimée à $23253 \mathrm{~km}^{2}$ soit $7 \%$ de la superficie totale de la Côte d'Ivoire qui est de 322462 km² (Ministère de l'environnement 2003 in N'Dri 2008). Le relief de la région de Fresco est parsemé de bas et de hauts plateaux, de plaines, de vallées et de bas-fonds marécageux alimentés par un important réseau hydrographique de rivières, fleuves (Bolo et Niouniourou) alimentant la lagune N'gni et qui à son tour atteint la mer par moment. Le littoral est la région la plus arrosée de la Côte d'Ivoire avec des précipitations annuelles supérieures à $1500 \mathrm{~mm}$. La zone qui abrite Fresco reçoit entre 1600 et 1800 $\mathrm{mm}$ par an; le climat reste pratiquement humide toute l'année (taux d'humidité $80 \%$ ), avec une température moyenne de $27^{\circ} \mathrm{C}$. Les sols sont hydromorphes plus ou moins humides adaptés à une végétation de forêt littorale associée aux groupements herbacés et fourrés littoraux. Les forêts marécageuses à dominante Mitragyna ciliata, Symphonia globulifera et Raphia abondent et les mangroves formées sur les sols hydromorphes à gley dans les estuaires sont dominées par les espèces arborées à palétuviers rouge Rhizophora racemosa et palétuviers blanc Avicennia germinans mais remplacées au fil du temps par une strate herbacée à Paspalum vaginatum ou arbustive à Drepanocarpus lunatus et Dalbergio ecastaphyllum (Egnankou, 2009). La forêt, presque entièrement détruite pour les cultures et la végétation, ne peut plus assurer totalement son rôle de protection des côtes et les mangroves détruites ne servent plus d'habitat privilégié pour de nombreuses espèces animales (poissons, crustacés, reptiles, oiseaux...). Le lamantin d'Afrique Trichechus senegalensis en voie de disparition vivant dans la lagune de Fresco fait l'objet de mesures de protection grâce au projet lamantin entrepris en collaboration avec la Wildlife Conservation Society (WCS) de New York depuis 1986. Ce projet couvre la région côtière qui s'étend de Grand-Lahou à Fresco.

\section{Géographie humaine}

Les populations autochtones qui occupent la zone de Fresco proviennent de la rencontre entre les deux grands groupes ethniques qui 
constituent les peuples du littoral : les Akans (les plus nombreux, côte est) et les Krous (en minorité, côte ouest). La langue parlée est le “Godié”. Le littoral ouest est peu peuplé contrairement à l'est qui présente une très forte population car les plus grands secteurs d'activité économique du pays sont regroupés autour d'Abidjan, la capitale économique du pays. A l'origine, le peuple Godié étant installé directement sur la côte entre la lagune et la mer vivait de la pêche principalement ; mais aujourd'hui, les populations s'adonnent à l'agriculture comme activité principale. La pêche est laissée aux étrangers dominés par les Fantis venus du Ghana (Fig 8a); ceux-ci se sentent lésés par la pêche moderne industrielle pratiquée à l'aide de chalutiers et produisant en très grande quantité. Les populations burkinabés, installées dans la région, constituent une main d'œuvre agricole très importante. Les boualés (peuples du centre de la Côte d'Ivoire) y habitent depuis de nombreuses années, dans des petits campements, et produisent de très grandes quantités de cacao $\left(1^{\mathrm{er}}\right.$ produit agricole de la région) et les autres cultures de rente telles que le café, l’hévéa, le palmier à huile, les noix de coco et aussi les produits vivriers (igname, riz, maïs, légumes, les agrumes). Le manioc, qui sert à la fabrication de l'attiéké, est produit continuellement constitue une source de revenue importante pour les femmes ; ce qui constitue au développement dans la région d'une activité commerciale intense. Quant à l'activité touristique, elle occupe une place moins importante car les plages de la zone de Fresco sont peu valorisées par rapport aux plages de la région sud-ouest (Monogaga, baie des sirènes à Grand-béréby) et donc moins fréquentées du fait de la vétusté des infrastructures de la ville et du mauvais état de la route qui rend son accès difficile.

\section{Cadre Geologique}

La géologie de la Côte d'Ivoire (Tastet et al. 1993, Mondé 1997, Sombo 2002, Digbéhi et al. 2011, Coulibaly et al.2012, Gnanzou 2014) révèle deux parties distinctes (i) un socle précambrien scindé en deux domaines : un domaine archéen (10\% du territoire) et un domaine protérozoïque (le reste du socle) dominé par les formations d’âge birrimien. Il est constitué de roches magmatiques (granites et granitoïdes, ...) et de roches métamorphiques (micaschistes, gneiss, migmatites, ...) parfois recoupées par des filons de roches basiques (dolérites); (ii) un bassin sédimentaire qui s'est formé au Jurassique supérieur au cours du rifting intracratonique et qui a séparé l'Afrique occidentale et l'Amérique latine lors de l'ouverture de l'Atlantique. Sa partie émergée représente 2,5\% (Fig 1) de la superficie du territoire et est traversée d'est en ouest par une importante faille «l'accident majeur des lagunes ». Celle-ci sépare : une partie nord avec une zone de couverture peu épaisse (hauts plateaux) constituée de sable, argile et grès plus ou moins ferrugineux (latérite) d'âges mio-pliocène et quaternaire et une partie sud où 
le bassin est subsident dans sa partie est, et où les formations sont dominées par des sédiments sablo-argileux marins d'âge crétacé supérieur au quaternaire qui représentent les bas plateaux et les plaines.

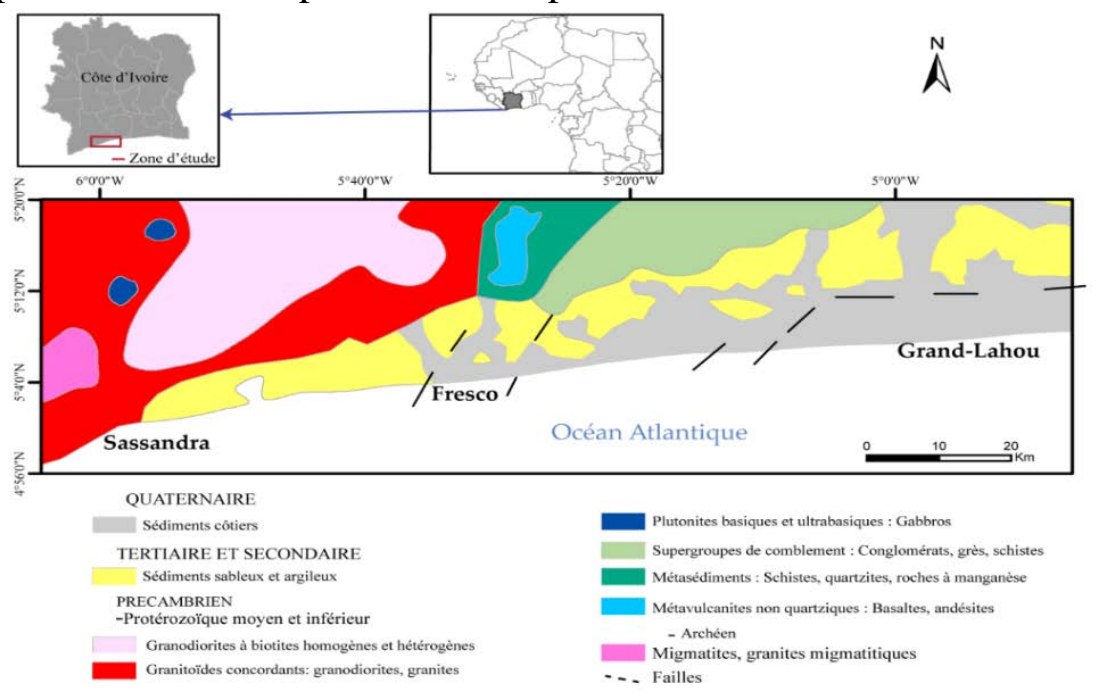

Figure 1. Carte géologique de la zone d'étude

Des études antérieures (Martin \& Tastet, 1972) ont indiqué que cet accident majeur serait accompagné de failles satellites qui lui sont parallèles et des failles méridiennes nord-sud. Ainsi, ces failles parallèles à la ligne de rivage sont probablement à l'origine de la formation des falaises vives de Fresco (Yao, 2012). En dehors de ces hautes falaises, on y trouve les bas plateaux formés de sables et d'argiles d'origine continentale de faible altitude (8 à $12 \mathrm{~m}$ environ). Ils forment un glacis sablo-argileux, aux pieds des hauts plateaux (Assemien et al., 1970), qui est considéré comme un épandage fluviatile de matériaux issus du continental terminal et des sables quaternaires. Les cordons sableux marins $(1,5$ à $6 \mathrm{~m})$ plus ou moins parallèles à la côte atteignent parfois des altitudes supérieures sur le littoral. Dans les affleurements des falaises fossilifères de Fresco apparaissent des formations d'argiles calcaires et gréseuses d'âge Paléocène-Eocène ; et qui leur confèrent une importance exceptionnelle à valoriser parmi les formations sédimentaires qui longent le littoral ivoirien.

\section{Approche Methodologique}

L’identification, des géosites potentiels d'une région donnée, suit une démarche systématique. Elle débute par un inventaire des sites d'intérêt géologique à travers une revue bibliographique des études déjà menées et des visites de terrain dans la zone concernée. Ensuite l'évaluation des sites identifiés se fait selon une analyse qualitative et quantitative en attribuant des notes pour chaque critère qui caractérise les éléments de valeur des différents 
sites d'intérêt. Pour qu'un site soit considéré comme définitif, c’est-à-dire un géosite, il est nécessaire que sa valeur soit élevée et justifiée sur la base de la note totale (moyenne) obtenue en faisant la somme des différentes notes attribuées par critère d'évaluation pour chaque élément qui compose ce site.

\section{Inventaire}

Selon Grandgirard (1999), réaliser un inventaire répond à trois objectifs : recenser, et classer des objets géologiques, mettre en exergue des éléments de valeur à des fins de sensibilisation et aussi pour des raisons de protection des sites géologiques. L'inventaire prend en compte également les éléments de la biodiversité; ainsi inventorier revient à identifier, recenser, dénombrer et classer les objets de la géodiversité et de la biodiversité susceptibles d'avoir une valeur particulière pouvant les intégrer dans le géopatrimoine. Dans le cas de cette étude, l'inventaire porte principalement sur les éléments de valeur qui caractérisent les falaises au plan scientifique. Ces falaises représentent le géosite principal le mieux connu qui caractérise la zone de Fresco. Ainsi, le but de cet inventaire est de faire l'analyse de détail du contenu des différentes strates (lithologie, fossiles, figures sédimentaires) qui permettra de les évaluer et de les présenter en tant qu'élément important de la géodiversité à valoriser sur divers plans : local, national et international comme patrimoine géologique.

\section{Evaluation scientifique}

Elle permet d'analyser qualitativement et quantitativement chaque géosite identifié à travers les critères choisis qui caractérisent les valeurs intrinsèques du géosite. Ces valeurs permettent de le distinguer comme géosite potentiel ou non. Selon Reynard et al. (2007), les éléments caractéristiques ou critères d'évaluation prennent en compte d'une part la valeur scientifique qui représente la valeur centrale et d'autre part les valeurs additionnelles (valeurs écologiques, esthétiques, culturelles et économiques). Pour l'évaluation scientifique, plusieurs auteurs se sont basés sur les trois critères principaux proposés par Grandgirard (1999) à savoir la rareté, l'intégrité et la représentativité puis l'ont adapté à chaque fois en fonction de l'échelle d'analyse et de l'objectif des projets d'étude (Reynard, 2004 ; Reynard et al. 2007; Poirier, 2008 ; Martin, 2011 ; Iosif, 2014 ; Reynard et al., 2016...). L'évaluation scientifique des falaises de Fresco s'appuie sur la méthode de Reynard et al. (2016). Celle-ci utilise comme critères: l'intégrité, la représentativité, la rareté et la valeur paléogéographique; chacun de ces critères est évalué indépendamment par un score numérique allant jusqu'à 1 (valeur plus élevée) par intervalle de 0,25. Les sites à grande valeur scientifique sélectionnés devraient être les meilleures de la région en ce qui 
concerne leur capacité à illustrer les processus et phénomènes géologiques visant la promotion des géosciences et devraient se présenter dans un bon état de conservation avec des caractéristiques qui les différencient des autres sites ayant des caractéristiques géologiques similaires (Reynard \& Brilha, 2018).

\section{Resultats: Valeur Scientifique Des Falaises Inventaire}

Les côtes qui bordent la partie sud de la ville de Fresco sont caractérisées par des falaises vives en contact permanent avec la mer. Ces falaises s'étendent sur près de $15 \mathrm{~km}$ de Fresco à Dassioko et sont connues pour leur richesse sédimentologique, paléontologique et stratigraphique.

Les eaux d'infiltration et l'effet de la houle qui vient battre régulièrement ces falaises, les exposent à l'érosion et entraînent des éboulements qui sont fréquents. Ainsi, les flancs décapés laissent entrevoir à travers les couches sédimentaires formées essentiellement de sables et d'argiles, de nombreuses figures sédimentaires (stratifications entrecroisées, stratifications obliques, grands chenaux, figures d'érosion) traduisant les mouvements eustatiques anciens ainsi que les processus sédimentaires qui y sont associés et révèlent l'histoire du bassin.

Certaines unités basales des falaises dans les argiles calcaires glauconieuses renferment un contenu fossilifère riche composé de nautiles, de foraminifères planctoniques et benthiques, des ostracodes, des gastéropodes, des discocyclines et des crustacés. Ces couches fossilifères sont connues comme les seules formations d'âge Paléocène-éocène qui affleurent dans le bassin sud-ouest ivoirien, ce qui leur confère une importance encore plus significative sur le plan scientifique. Cette richesse que révèlent les falaises de Fresco justifie les nombreuses missions d'études géologiques qui y sont organisées chaque année par les universités et les grandes écoles ivoiriennes notamment l'Unité de Formation et de Recherches des Sciences de la Terre et des Ressources Minières (UFR- STRM) de l'université Félix Houphouët Boigny, l'Institut National Polytechnique Houphouët Boigny (INPHB), les groupes LOKO, l'Ecole Supérieure des Bâtiments et Travaux Publics (ESBTP), etc. Ces falaises s'étendent de Fresco à Dassioko et peuvent atteindre des altitudes allant de moins de $20 \mathrm{~m}$ à près de $30 \mathrm{~m}$ environ ; on les regroupe en cinq falaises :

Tableau 1. Localisation des falaises de Fresco

\begin{tabular}{|c|c|c|c|}
\hline Falaises & Code & Hauteur & Coordonnées \\
\hline Falaise de Françoikro & FAL01 & $18 \mathrm{~m}$ & N0504’37 / W05³6’09 \\
\hline Falaise de 12 Frères & FAL02 & $22,5 \mathrm{~m}$ & 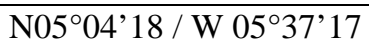 \\
\hline Falaise de Fanti & FAL03 & $21 \mathrm{~m}$ & $\mathrm{~N}^{2} 5^{\circ} 03^{\prime} 32 / \mathrm{W} 05^{\circ} 42^{\prime} 31$ \\
\hline Falaise Kossô & FAL04 & $20 \mathrm{~m}$ & 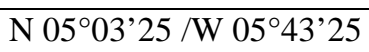 \\
\hline Falaise de Dassioko & FAL05 & $19 \mathrm{~m}$ & 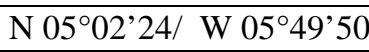 \\
\hline
\end{tabular}




\section{Falaise de Françoikro}

C'est la première falaise que l'on rencontre lorsqu'on part de l'est vers l'ouest en suivant la côte. D'une hauteur de $18 \mathrm{~m}$ environ, elle présente à sa base une couche d'argiles calcaires très fossilifères avec de nombreux minéraux de glauconite et quelques minéraux de pyrite (Fig 2a). Son contenu fossilifère justifie l'intérêt porté aux falaises de Fresco et les nombreuses études qui y ont été menées. La datation basée sur l'identification des faciès de microfossiles a permis de donner l'âge Eocène inférieur (Yprésien) à cette formation basale dans la falaise de Françoikro (Yao, 2012). Le bassin sédimentaire ivoirien s'est formé au Crétacé et présente des formations carbonatées rares. Au niveau de Fresco, elles apparaissent dans les argiles lumachelliques des falaises de Françoikro et de 12 Frères; et sont dues à l'abondance des fossiles à tests calcaires qui se sont désagrégés et accumulés dans les sédiments. Les stratifications entrecroisées et ondulées observées dans ces lumachelles (Fig 2b) indiquent que ces argiles calcaires plus ou moins sableuses se sont déposées dans un environnement sédimentaire agité. La numérotation des couches de $\mathrm{E}$ à $\mathrm{H}$ est dû au fait que les formations de cette falaise correspondent aux couches supérieures de la falaise de 12 Frères plus haute et renferme la totalité des couches contenues dans les falaises.

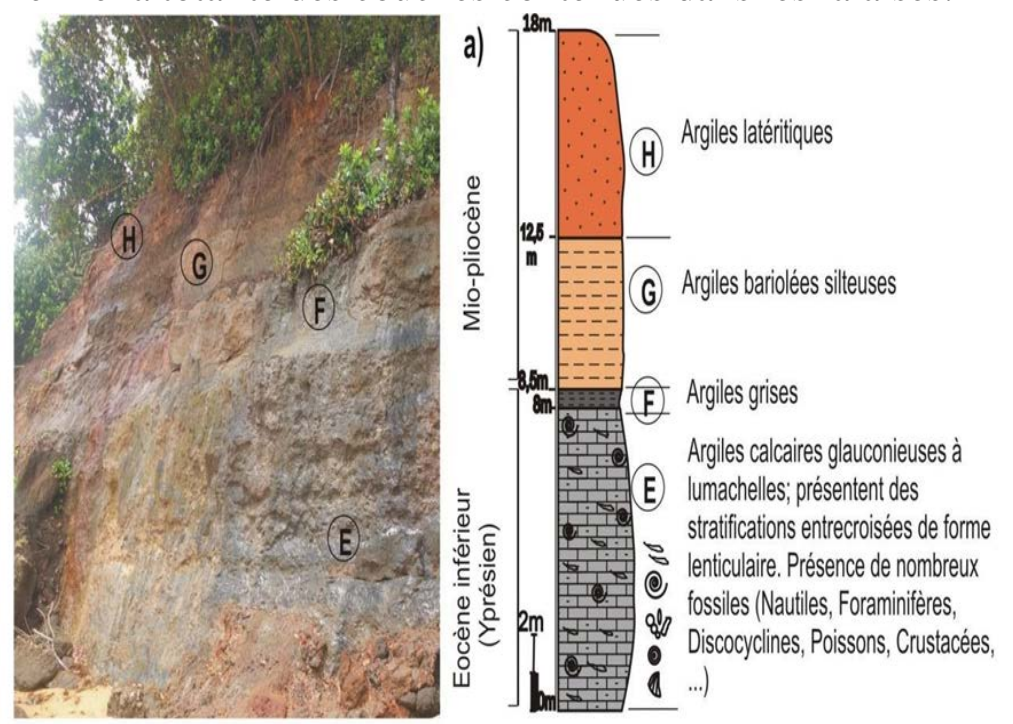




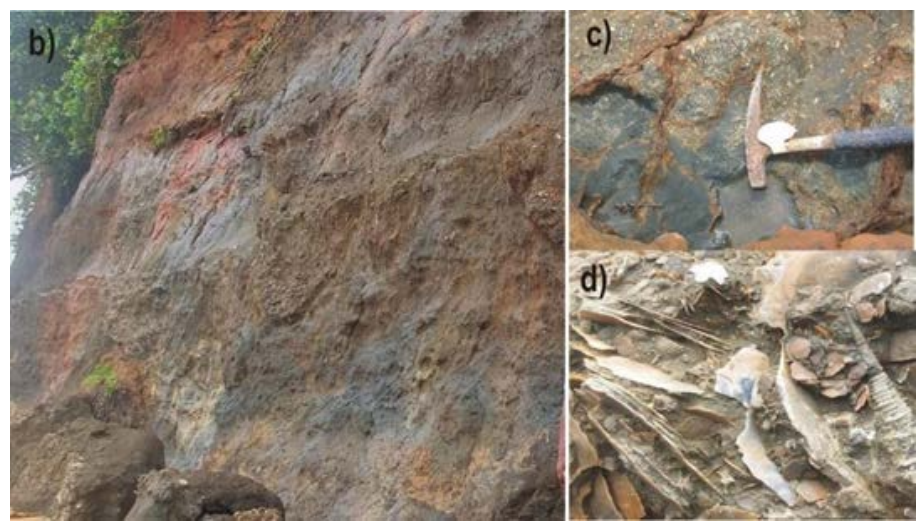

Figure 2. a) Log stratigraphique de la Falaise de Françoikro ; b) Stratifications entrecroisées, lenticulaires ; c) Argiles calcaires glauconieux verdâtre montrant de fines lumachelles ; d) Grands discocyclines et gastéropodes

\section{Falaise de 12 Frères}

Elle renferme la totalité des couches sédimentaires rencontrées dans les falaises et numérotées de A à H (Fig 3a). C’est la falaise la plus haute, pouvant atteindre $25 \mathrm{~m}$.

Elle présente également la couche argileuse fossilifère observée dans la première falaise, mais avec une épaisseur moindre $(5 \mathrm{~m})$ et inclinée formant un biseau dans sa partie ouest. Dans les argiles latéritiques s'observe une figure sédimentaire spectaculaire qui caractérise la falaise des 12 Frères. Il s'agit d'un grand chenal dont le comblement s'est fait par des argiles fines de sables et de galets subanguleux de tailles variables dans un désordre sédimentaire traduisant un environnement de dépôt très agité (Fig 3b). Ces latérites renferment de nombreuses traces de bioturbation liées à l'activité des crustacés, des poissons et de divers foraminifères (Fig 4c). Le niveau lenticulaire d'argile calcaire lumachellique très fossilifère présente des stratifications entrecroisées obliques (Fig 2b). Cette couche a une coloration qui varie du gris foncé au vert noirâtre du fait de la présence de la glauconite ; on y trouve également les minéraux de pyrite mais moins abondants. Elle est très riche en fossiles et comprend de grands foraminifères (Fig 2c $-2 d$ ): nautiles (Deltoïdonautilus frescoensis); discocyclines (Discocycline archiaci, Discocycline fortisi ...) et nummulites et de petits foraminifères : foraminifères planctoniques (Morozovella acuta, Acarinina primitiva, Planorotalites chapmani ...); foraminifères benthiques (Lenticulina sp, Anomaloïdes sp ...) et ostracodes (Hermanites sp ; Reticulin sp ...). 

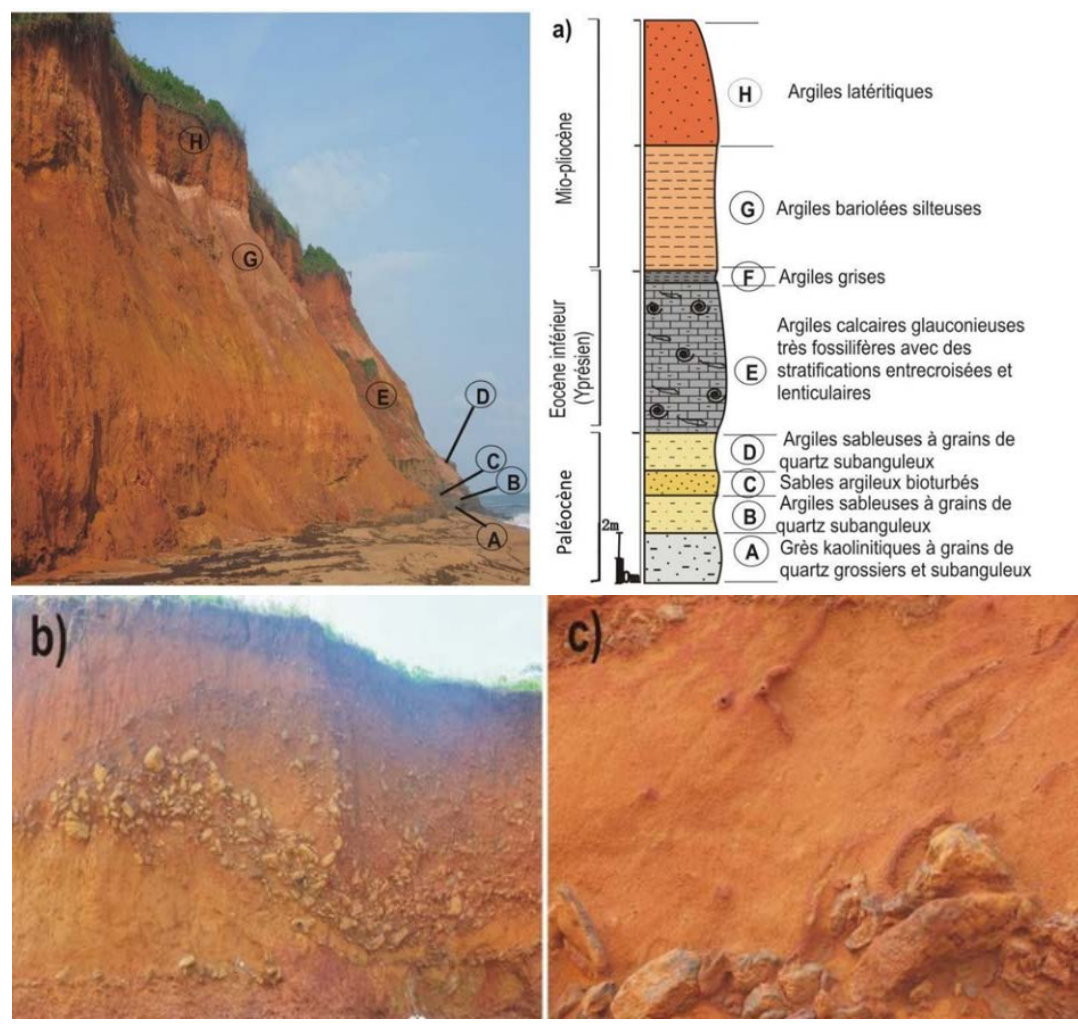

Figure 3. a/ Log stratigraphique de la falaise de 12 Frères ; b/ Grand chenal formant un biseau ; c/ Traces de bioturbation

\section{Falaise de Fanti}

Dans cette falaise, la couche lenticulaire d'argiles calcaires glauconieuses et absente et les couches supérieures (argiles ferrugineuses) du Mio-pliocène reposent directement sur les formations paléocènes. Cette absence est due à sa position surélevée par rapport aux deux premières falaises et traduit donc une phase de non dépôt de ces sédiments fossilifères (Fig 4a). Comme figures sédimentaires l'on remarque à la base, des figures de charges dans les grès et de nombreuses traces de bioturbation (Fig 4b). La présence de hardground traduit un arrêt de sédimentation et justifierait une régression marine; les eaux d'infiltration créent le lessivage des couches de dessus et entraînent les minéraux de fer donnant une coloration ocre dans les grès kaolinitiques blanchâtres (Fig 4c). 

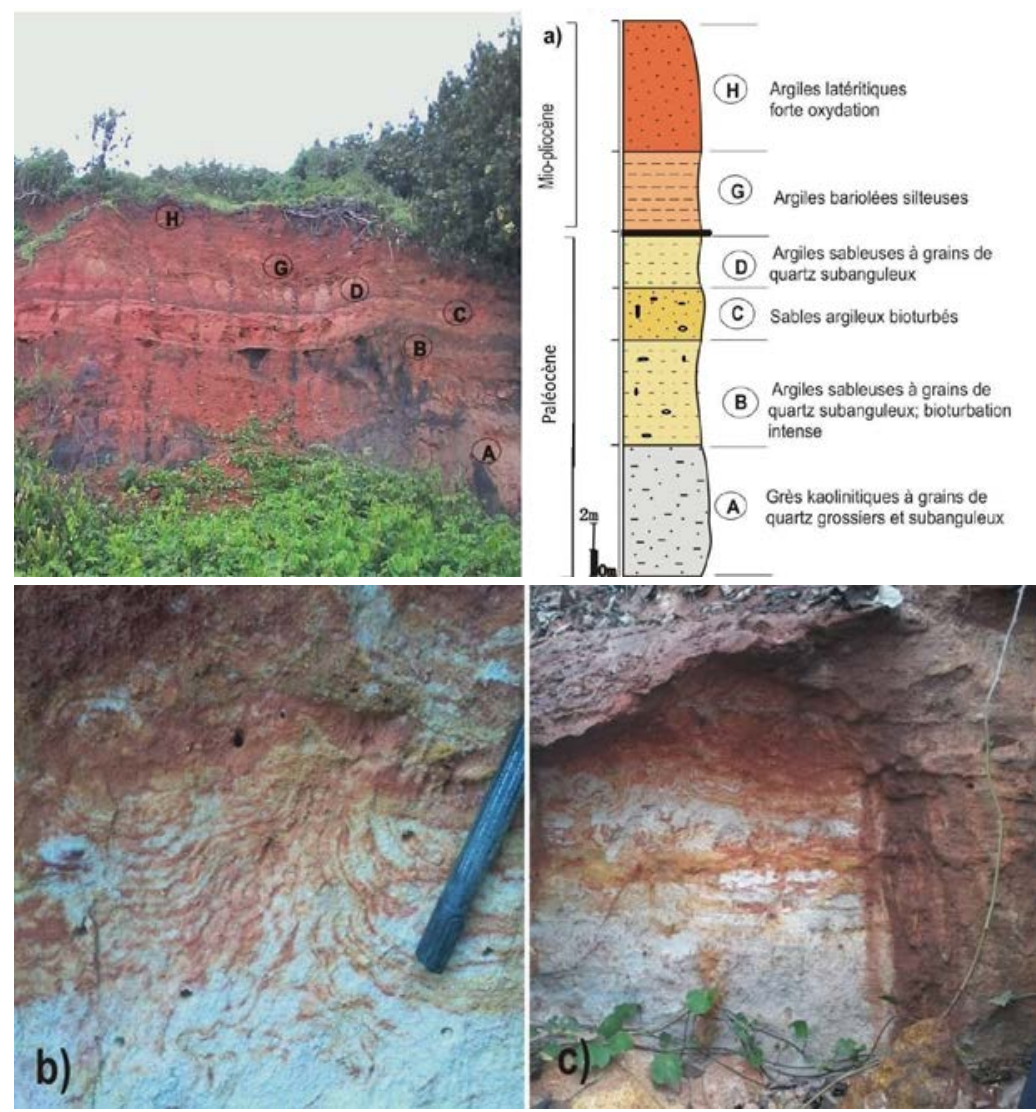

Figure 4. a) Log stratigraphique de la Falaise de Fanti ; b - c) Figures de charge ; Oxydation du grès kaolinitique

\section{Falaise de Kossô}

La falaise de Kossô est caractérisée par de grandes stratifications obliques formant des chenaux entrecroisés. Ceci explique que les sédiments argileux se sont déposés dans un environnement plus ou moins calme avec des phases d'érosions successives. Les stratifications obliques forment des lentilles de tailles variables dans des argiles sableuses fines (Fig 5a). Cette falaise présente particulièrement plusieurs hardgrounds. Ces surfaces encroutées d'oxydes de fer et de manganèse traduisent un arrêt dans la continuité de la sédimentation (discordance lacunaire). Les sédiments de la falaise de Kossô sont fortement oxydés et cela se traduit par la leur coloration qui prend plusieurs teintes (rouge, ocre, orange, jaune, violet...) du fait des minéraux de Fer, Aluminium, et de Manganèse très abondants (Fig 5b). 

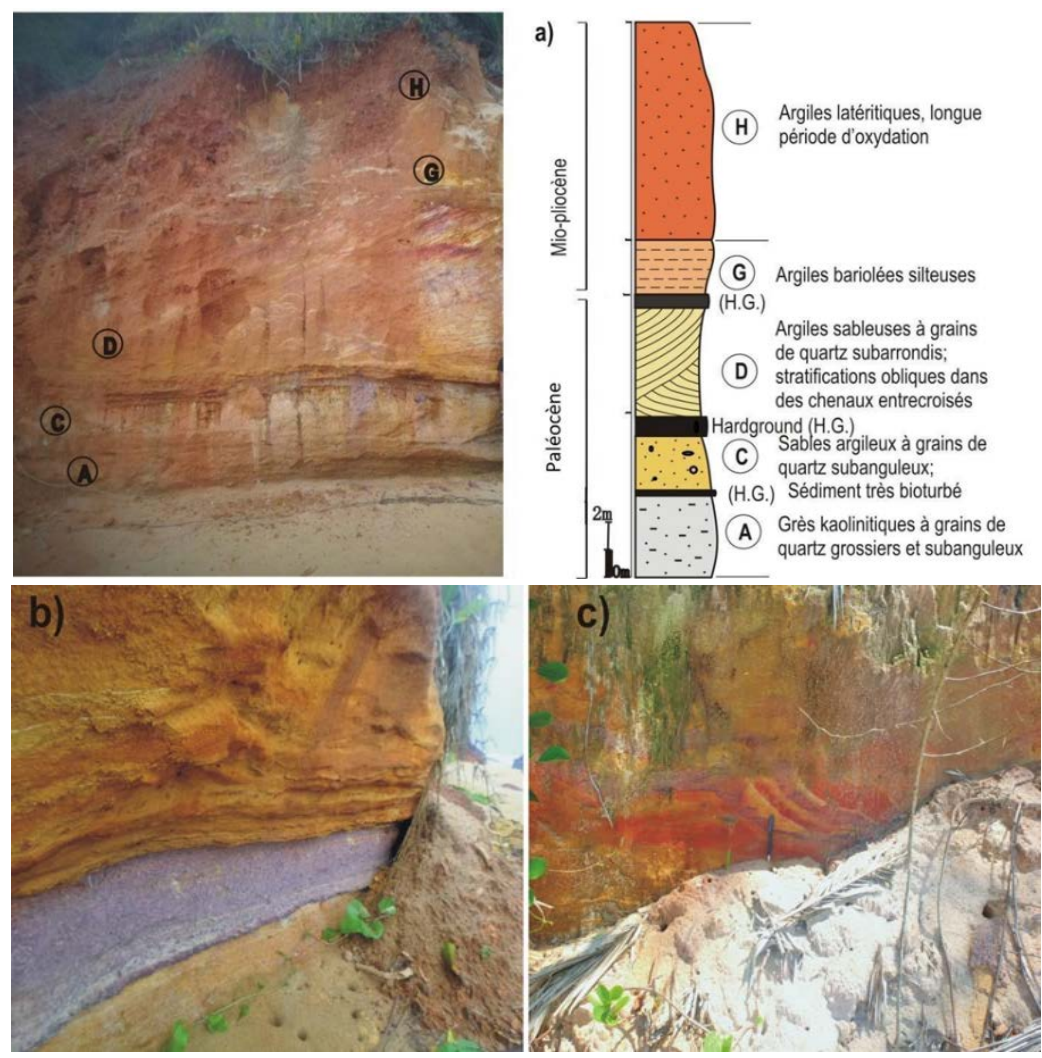

Figure 5 . a) Log stratigraphique de la Falaise de Kossô; b-c) Diverses colorations des sédiments oxydés

\section{Falaise de Dassioko}

Cette falaise subit fortement l'érosion de la houle. Ses formations argileuses moins dures (argiles sableuses) sont constamment en éboulement. Elles forment des encoches dans la falaise, rendant cette partie encore plus vulnérable à l'éboulement (Fig 6a). C'est la plus extrême à l'ouest et les minéraux de pyrite très abondants, les sables grossiers quartzeux anguleux, et les galets de quartz subanguleux à anguleux traduisent la proximité du socle (Fig 6b-6c). L'altération du socle précambrien, l'action des vagues sur ces sédiments issus de cette altération complétée du matériel sédimentaire charrié par les cours d'eau a fourni l'essentiel des sédiments ayant formé les falaises de Fresco. 

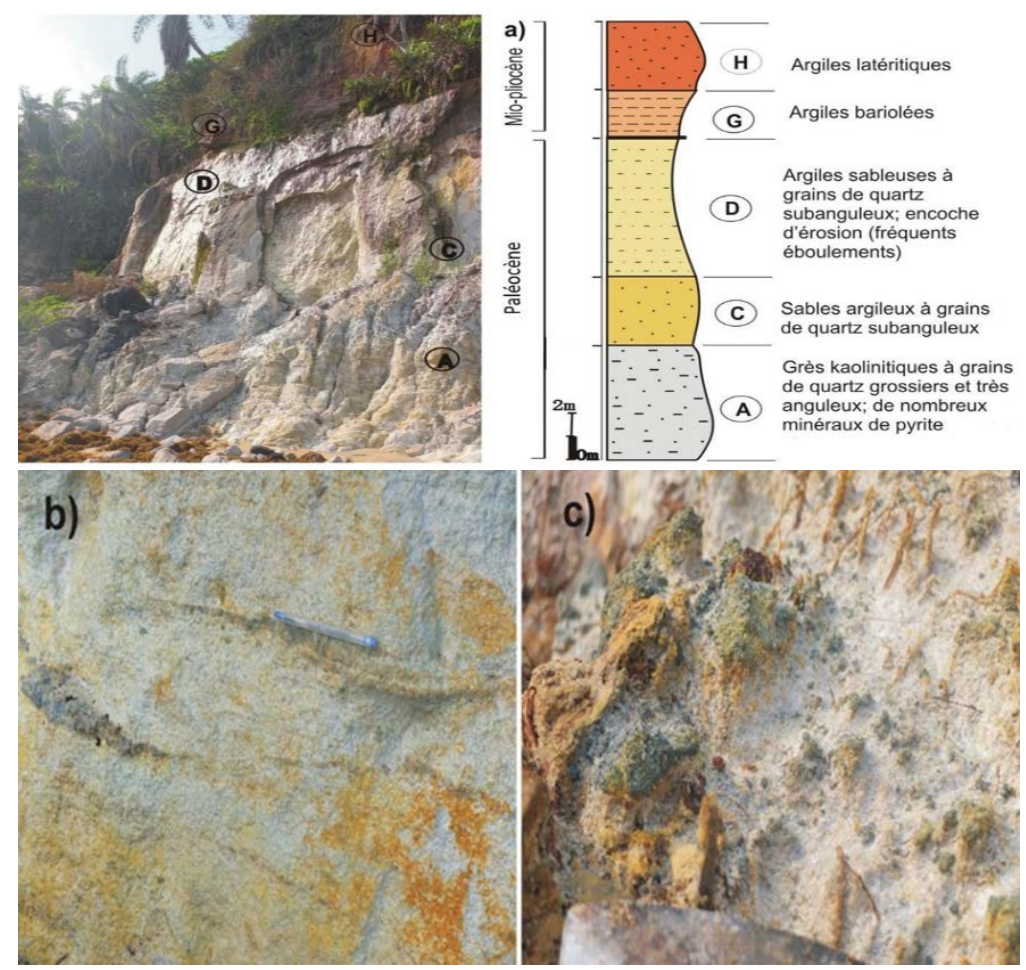

Figure 6. a) Log stratigraphique de la Falaise de Dassioko ; b) Grès kaolinitique à grains de quartz grossiers et anguleux ; c) Minéraux de pyrite

\section{Evaluation scientifique}

L'évaluation scientifique des falaises à partir des scores attribués pour chaque critère d'évaluation permet d'en relever les valeurs exceptionnelles qui sont résumées dans les grilles d'évaluation suivantes (Tab 2à 6):

Tableau 2. Résultats de l'évaluation scientifique de la Falaise de Françoikro

\begin{tabular}{|c|c|c|}
\hline Géosites & Critères d'évaluation & Notes \\
\hline \multirow{4}{*}{$\begin{array}{c}\text { Falaise de } \\
\text { Françoikro } \\
\text { FAL(01) }\end{array}$} & Intégrité: La falaise présente des zones de forts éboulements & 0,5 \\
\hline & $\begin{array}{l}\text { Représentativité: Le contenu fossilifère a permis la datation précise } \\
\text { des formations sédimentaires de la région, et de voir plusieurs } \\
\text { fossiles à l'affleurement. }\end{array}$ & 1 \\
\hline & $\begin{array}{l}\text { Rareté: Elle renferme la couche importante d'argile glauconieuse } \\
\text { (11 m) et fossilifère d'âge Paléocène-éocène connue à Fresco } \\
\text { (Tessier } 1960 \text {; Tastet et al.1993 ; Yao 2012). }\end{array}$ & 1 \\
\hline & $\begin{array}{c}\text { Valeur paléogéographique: Elle traduit les environnements de } \\
\text { dépôts des sédiments de la région et les mouvements eustatiques } \\
\text { anciens à travers les figures sédimentaires observées. }\end{array}$ & 0,75 \\
\hline \multicolumn{2}{|r|}{ Valeur scientifique Falaise de Françoikro } & $0,812 / 1$ \\
\hline
\end{tabular}


Tableau 3. Résultats de l'évaluation scientifique de la falaise des 12 Frères

\begin{tabular}{|c|c|c|}
\hline Géosites & Critères d'évaluation & Notes \\
\hline \multirow{3}{*}{$\begin{array}{c}\text { Falaise de 12 } \\
\text { Frères }\end{array}$} & $\begin{array}{c}\text { Intégrité: La falaise est conservée mais présente des éboulis à la } \\
\text { base. }\end{array}$ & $\mathbf{0 , 5}$ \\
\cline { 2 - 3 } & $\begin{array}{c}\text { Représentativité: Elle est la plus haute de toutes et renferme la } \\
\text { totalité des couches sédimentaires et a permis de préciser l’âge des } \\
\text { formations (Yao 2012). }\end{array}$ & $\mathbf{0 , 7 5}$ \\
\cline { 2 - 3 } & $\begin{array}{c}\text { Rareté: C’est la seule qui renferme toutes les couches des } \\
\text { formations sédimentaires rencontrées. }\end{array}$ & $\mathbf{1}$ \\
\cline { 2 - 3 } & $\begin{array}{c}\text { Valeur paléogéographique : Elle traduit les environnements de } \\
\text { dépôts des sédiments de la région ainsi que les mouvements } \\
\text { eustatiques anciens à travers les figures sédimentaires observées. }\end{array}$ & $\mathbf{0 , 7 5}$ \\
\hline \multicolumn{2}{|c|}{ Valeur scientifique Falaise de 12 Frères } & $\mathbf{0 , 7 5 / 1}$ \\
\hline
\end{tabular}

Tableau 4. Résultats de l'évaluation scientifique de la falaise de Fanti

\begin{tabular}{|c|c|c|}
\hline Géosites & Critères d'évaluation & Notes \\
\hline \multirow{4}{*}{$\begin{array}{c}\text { Falaise de } \\
\text { Fanti }\end{array}$} & $\begin{array}{c}\text { Intégrité: La falaise est affectée par l'érosion et présente des zones } \\
\text { d'éboulement }\end{array}$ & $\mathbf{0 , 5}$ \\
\cline { 2 - 3 } & $\begin{array}{c}\text { Représentativité: Les formations de cette falaise ont servi par } \\
\text { corrélation avec les sondages à confirmer l'âge des niveaux. }\end{array}$ & $\mathbf{0 , 7 5}$ \\
\cline { 2 - 3 } & $\begin{array}{c}\text { Rareté: Elle comporte un niveau bitumineux qui suscite la curiosité } \\
\text { sur l'origine de ce dernier ; l'absence de la couche d'argiles calcaires } \\
\text { glauconieuses et fossilifère dans cette falaise confirme sa forme en } \\
\text { biseau. }\end{array}$ & $\mathbf{0 , 7 5}$ \\
\cline { 2 - 3 } & $\begin{array}{c}\text { Valeur paléogéographique: Elle traduit les environnements de } \\
\text { dépôts des sédiments de la région ainsi que les mouvements } \\
\text { eustatiques anciens à travers les figures sédimentaires observées. }\end{array}$ & $\mathbf{0 , 7 5}$ \\
\hline & Valeur scientifique Falaise de Fanti & $\mathbf{0 , 6 8 7 / 1}$ \\
\hline
\end{tabular}

Tableau 5. Résultats de l'évaluation scientifique de la falaise de Kossô

\begin{tabular}{|c|c|c|}
\hline Géosites & Critères d'évaluation & Notes \\
\hline \multirow{3}{*}{$\begin{array}{c}\text { Falaise de } \\
\text { Kossô }\end{array}$} & $\begin{array}{c}\text { Intégrité: La falaise est en éboulement et les formations sont } \\
\text { affectées par une forte oxydation }\end{array}$ & $\mathbf{0 , 5}$ \\
\cline { 2 - 3 } FAL(04) & $\begin{array}{c}\text { Représentativité: Cette falaise présente des stratifications obliques } \\
\text { dans des chenaux entrecoupés qui traduisent les mouvements } \\
\text { eustatiques anciens. }\end{array}$ & $\mathbf{0 , 7 5}$ \\
\cline { 2 - 3 } & $\begin{array}{c}\text { Rareté: Elle permet une meilleure explication des mouvements } \\
\text { eustatiques successifs anciens. }\end{array}$ & $\mathbf{0 , 7 5}$ \\
\cline { 2 - 3 } & $\begin{array}{c}\text { Valeur paléogéographique: Elle traduit les environnements de } \\
\text { dépôts des sédiments de la région ainsi que les mouvements } \\
\text { eustatiques anciens à travers les figures sédimentaires observés. }\end{array}$ & $\mathbf{0 , 7 5}$ \\
\hline & Valeur scientifique Falaise de Kossô & $\mathbf{0 , 6 8 7 / 1}$ \\
\hline
\end{tabular}


Tableau 6. Résultats de l'évaluation scientifique de la Falaise de Dassioko

\begin{tabular}{|c|c|c|}
\hline Géosites & Critères d'évaluation & Notes \\
\hline \multirow{3}{*}{$\begin{array}{c}\text { Falaise de } \\
\text { Dassioko } \\
\text { FAL(05) }\end{array}$} & $\begin{array}{c}\text { Intégrité: La falaise présente de fréquents éboulements à divers } \\
\text { endroits }\end{array}$ & $\mathbf{0 , 5}$ \\
\cline { 2 - 3 } & $\begin{array}{c}\text { Représentativité: Les grains grossiers et très anguleux, les nombreux } \\
\text { minéraux de pyrite traduisent la proximité du socle }\end{array}$ & $\mathbf{0 , 5}$ \\
\cline { 2 - 3 } & $\begin{array}{c}\text { Rareté: La falaise est la plus extrême à l'ouest et confirme la présence } \\
\text { du socle à mesure que l'on s'avance vers Sassandra. }\end{array}$ & $\mathbf{0 , 5}$ \\
\cline { 2 - 3 } & $\begin{array}{c}\text { Valeur paléogéographique: Elle traduit les environnements de } \\
\text { dépôts des sédiments de la région et les mouvements eustatiques } \\
\text { anciens à travers les figures sédimentaires observées. }\end{array}$ & $\mathbf{0 , 7 5}$ \\
\hline & Valeur scientifique Falaise de Dassioko & $\mathbf{0 , 5 6 2 / 1}$ \\
\hline
\end{tabular}

\section{Synthèse}

L'évaluation scientifique des falaises indique que chacune d'elle affiche une note supérieure à la moyenne $(0,5)$; ce qui signifie qu'elles présentent toutes un intérêt scientifique particulier. Les falaises de Françoikro et des 12 Frères présentent les valeurs les plus élevées et qui sont respectivement de 0,812 et 0,75 . Cela est dû au fait qu'elles contiennent la plus importante couche, celle d'argile calcaire et fossilifère qui confère un grand intérêt scientifique des falaises. Ces argiles calcaires glauconieuses riches en fossiles (foraminifères planctoniques et benthiques, ostracodes) ont permis, avec des données de deux sondages de dater et d’établir la stratigraphie des formations de la zone (Yao, 2012) puis relater l'histoire des falaises. Les fréquents éboulements dus à l'érosion ont justifié la valeur moyenne de 0,5 relative à l'intégrité des falaises. L'évaluation des falaises de Fanti et de Kossô fournit une même moyenne 0,687 bien qu’elles présentent des différences au niveau de leurs caractéristiques. Les figures sédimentaires (hardgrounds, chenaux entrecroisés) présentes dans la falaise de Kossô décrivent les mouvements eustatiques et permettent d'interpréter les environnements de dépôt des formations. La falaise de Dassioko présente la note la moins élevée d'une valeur de 0,562 car sur le plan scientifique elle fournit peu d'informations sur les conditions de mise en place des falaises étudiées. La valeur scientifique globale de l'ensemble des géosites des falaises est de 0,7/1 et confirme donc leur grande importance et leur utilité pour les travaux de recherches géologique et la nécessité de leur accorder un intérêt particulier en tant que géosites potentiels non seulement de la région sud-ouest, mais aussi au niveau national voire international.

\section{Environnement naturel du géosite}

La côte sud-ouest est caractérisée par son relief accidenté de hauts plateaux en «dents de scie ». Ces hauts plateaux atteignent des altitudes plus élevées entre 50 et $100 \mathrm{~m}$ vers l'ouest dans les régions de San-pedro et Monogaga. A Fresco ces plateaux sont moins élevés (20-50 m) forment avec 
des collines des vallées plus ou moins élargies, empruntées par un important réseau hydrographique de fleuves et de rivières et lagunes. Ces cours d'eau créent avec les nombreuses précipitations que connaît la région un climat qui reste humide toute l'année. Aussi la végétation de forêt littorale, de savanes herbacées autour des mangroves est adaptée à ce climat tropical humide. En effet, en quittant la ville de Fresco, on traverse la lagune puis on passe par la forêt et les collines et vallées couvertes de végétation représentant la zone de transition entre le continent et la mer, pour atteindre les falaises qui la bordent. Cet ensemble forme un milieu naturel très agréable (Figure 7) et favorable pour de nombreuses espèces animales telles que les mammifères (singes, rongeurs, éléphants...) ; les oiseaux (hirondelles, pigeons, pintades, rapaces...); les reptiles (crocodiles, serpents, tortues, lézards ...) et amphibiens. Les eaux sont riches en poissons (Tilapia, Capitaine, Mérou, Hareng, Raie...) et crustacés (crabes, langoustes, ...) et gastéropodes (g. Pachymelania) qui se réfugient préférentiellement dans les racines échasses des palétuviers qui y sont implantés. Ces eaux abritent particulièrement le lamantin d'Afrique, mammifère spécifique de l'écosystème lagunaire et des estuaires de Fresco, faisait autrefois l'objet de visite de nombreux touristes tout comme les hautes falaises qui représentent les escarpements des hauts plateaux en face de la mer, elle-même un véritable atout pour la région.

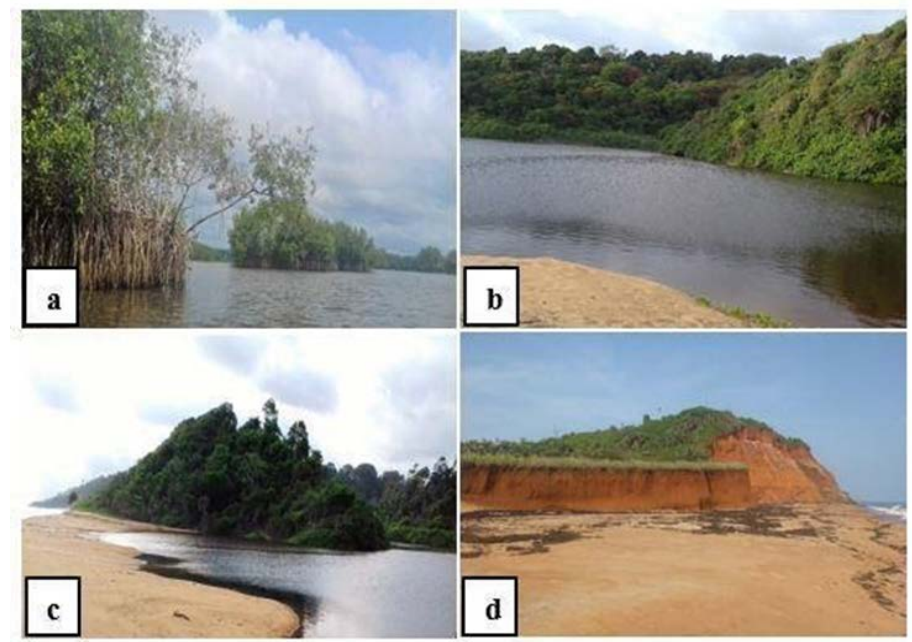

Figure 7. Environnement naturel de Fresco ; a) Lagune de Fresco présentant des palétuviers ; b) Forêt littorale ; c) Estuaire ; d) Falaise

\section{Activités socio-économique, culturelle et touristique}

L’Autorité pour l'Aménagement de la Région du Sud-Ouest (ARSO), société de développement à compétence régionale placée sous la tutelle directe de la présidence de République a été créée le 22 Décembre 1969. L'amélioration du tronçon Abidjan-San Pedro, la construction de port de San 
Pedro et l'avènement de l'agro-industrie ont favorisé l'évolution sur le plan agraire, urbain, industriel et touristique de la région (N’Dri, 2008); plus particulièrement dans les villes de Sassandra, San Pedro, Grand-Béréby et Tabou pour leurs belles baies et plages sablonneuses à côté des caps rocheux. L'eau de mer présente une couleur plus claire qui varie du bleu au vert montrant l'aspect naturel et préservé du milieu ; ceci permet aux touristes de bénéficier d'un cadre paisible de détente et de loisir. L'activité touristique était principalement portée sur le tourisme balnéaire, et la ville de Fresco bien qu'ayant les mêmes atouts naturels ne bénéficiait pas d'autant d'attention. Mais était connue particulièrement pour ses hautes falaises sédimentaires et sa lagune abritant une importante quantité de lamantins. Cependant, les populations locales profitent de ces richesses à travers l'activité agricole (café, cacao, palmier à huile, vivriers, ..), la pêche, et le commerce. Mais celles-ci rencontrent des difficultés pour acheminer et vendre leurs produits car les lieux de production sont éloignés des voies principales, pas de routes bien tracées accessibles par les véhicules et les pistes sont souvent impraticables. Comme activités récréatives elles pratiquent la baignade, la pêche traditionnelle, les courses à pirogue, les festivals culinaires entre villages. Le tourisme a toujours été pratiqué d'une manière lente dans le sud-ouest contrairement à la côte est qui est plus peuplée et dotée d'énormes d'infrastructures (grands complexes hôteliers, nombreux restaurants, musé de civilisation ...), bien entretenus; ceci a été encore plus accentué par la forte dégradation de la route côtière mais aussi par les crises politiques débutées en 1999 qui ont fait baisser la notoriété de la Côte d'Ivoire au plan international. A Fresco, le tourisme est pratiqué de façon occasionnelle et les visites concernent des excursions à pied (randonnée dans la forêt, visite des falaises, balade sur la plage) (Fig 8) ou à pirogue sur la lagune pour voir les îles de mangrove ou quelques rares lamantins qui y vivent encore. Cette activité est peu rémunératrice pour les fils de la région qui préfèrent s'adonner à leurs activités habituelles. Notons cependant que la tradition Godié joue un rôle capital dans l'attrait touristique à travers des chants et des danses traditionnelles qui attirent beaucoup de spectateurs, le festival Pôpaix, la promotion des danses du terroir telles que l'Abi Ayara de Dadjeré, l'Alloukou, le Sakékowê (danse du Grélot) et la mythique danse Tosi des femmes du village Guédikpô. Fresco manque énormément de structures d'accueil et a besoin de réhabiliter les anciens édifices et construire de nouvelles infrastructures en vue de réactiver la splendeur de la ville et attirer plus de touristes pour accéder au développement socio-économique de la région ; et cela doit être précédé de la réhabilitation de la route côtière principale. Depuis quelques années les autorités régionales s'y activent ardemment et dans cette optique s'est tenu en Octobre 2017 à Palm Club Hôtel à Abidjan un atelier avec les partenaires au développement (WABiCC-USAID) pour identifier les menaces, les risques, les vulnérabilités 
et les opportunités socio-économiques et proposer des solutions d'adaptation pour la ville de Fresco.

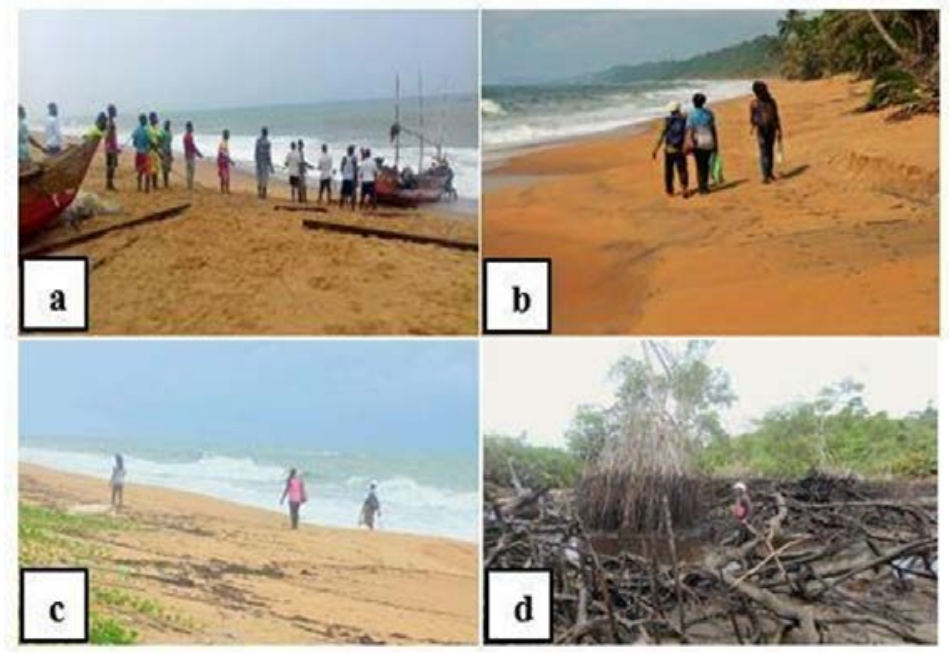

Figure 8. a) Pêche pratiquée en mer par les Fanti; b - c)Visites guidées sur la plage ; d) Destruction de mangrove par l'ouverture de l'embouchure (technique de pêche artisanale)

\section{Discussion Et Conclusion}

L'évaluation scientifique des falaises réalisée dans cette étude montre qu'elles ont une intégrité et des valeurs paléogéographiques similaires. Cependant, elles présentent chacune une certaine rareté et sont représentatives dans la caractérisation des milieux de dépôt des formations de la région de Fresco. La grande importance des falaises de Fresco se rapporte principalement à leur intérêt paléontologique du fait des nombreux fossiles ayant permis d'identifier avec précision l’âge Eocène inférieur (Yprésien) des argiles calcaires glauconieuses et identifier des grès kaolinitiques et argiles sableuses d'âge Paléocène à la base des falaises. Des marnes et calcaires à faune de mollusques et discocyclines et de nautiles observés dans la falaise de Thiès au Sénégal sont également d’âge Yprésien (Tessier 1952). Les formations fossilifères dans les falaises de Fresco présentent de grandes stratifications entrecroisées traduisant d'anciennes dunes sous-marines tout comme celles des falaises calcaires de Bonifacio et dans les carrières d'Orival (Jonin et al. 2007) ; mais celles-ci présentent des dunes bien visibles exondées à l'issue d'une longue histoire géologique. L'ensemble de ces objets géologiques remarquables (formations fossilifères, figures sédimentaires) représente des témoins des paléo-milieux ayant permis de reconstituer le paléoenvironnement lors de la mise en place des falaises tout en précisant les variations du niveau de la mer. Ces géosites des falaises de Fresco deviennent donc un repère stratigraphique régional à partir duquel l'on peut identifier par des corrélations les autres formations du bassin sédimentaire. Ceci traduit sa 
valeur exceptionnelle au de vue de leur rareté en tant que géosites potentiels du patrimoine géologique en Côte d'Ivoire. Il serait judicieux d'exposer les spécimens déjà collectionnés (nautiles de Fresco) dans un musée proche des sites de prélèvement que sont les falaises; tout comme fut le cas pour le géosite du «mur des douaniers » dans la réserve naturelle de Vireux-Molhain en France. Ce gisement paléontologique connu pour sa riche faune de trilobites a fournis de nombreux spécimens qui sont bien conservés et exposés à Bognysur-Meuse ; c'est une richesse patrimoniale conservée ex-situ (Jonin et al. 2007). Cette étude aura permis de montrer la valeur scientifique et pédagogique des falaises de Fresco à travers un inventaire détaillé et une évaluation des formations sur les plans sédimentologique et paléontologique. Aussi, la présentation d'une biodiversité riche qui entoure ces falaises permet de montrer l'état de conservation de la zone malgré l'influence anthropique par endroits. L'étude de ces falaises comme premier site du géopatrimoine révèle le contexte d'adhésion de la Côte d'Ivoire dans le Réseau Africain de Géoparc (AGN), mais aussi permet d'attirer l'attention des autorités locales sur l'importance de ces géosites des falaises en tant qu' éléments importants du géopatrimoine, qui aura besoin d'une meilleure promotion à travers une valorisation (piste bien tracées, panneaux indicateurs ...) en vue de favoriser le géotourisme pouvant contribuer au développement durable et socioéconomique de la région de Fresco.

\section{References:}

1. Assemien P., Filleron J.C., Martin L., Tastet J.P. (1970) Le Quaternaire de la zone littorale de Côte d'Ivoire. Ass. Sénégal. Et. Quatern. Ouest afr., Bull. Liaison, Sénégal, n² 25, pp. 65-78.

2. Brilha J. (2016): Inventory and Quantitative Assessment of Geosites and Geodiversity Sites: a review Geoheritage, v. 8, no. 2, pp. 119-134.

3. Coulibaly, Y., Kouaho, B., Gnanzou, A., Allialy, M.E. et Djro, S.C. (2012). Contexte géologique de la minéralisation aurifère du prospect de Bobosso (région de Dabakala, centre-nord de la Côte d'Ivoire). J. Rech. Sci. Univ. Lomé (Togo), 2012, Série A, 14(2): 149-162

4. Digbehi Z. B., Toé Bi K. K., Adopo K. L., Guédé K. E., Tahi I. et Yao K. R. (2011) : Palynologie et environnements de dépôt des sédiments d'âge cénomanien supérieur-maastrichtien inférieur dans le bassin offshore de Côte d'Ivoire (Afrique de l'ouest). Sciences \& Nature Vol. $8 \mathrm{~N}^{\circ} 1$, p 95 - 105

5. Egnankou W. M. (2009) Réhabilitation des mangroves comprises entre Fresco et Grand-Lahou en Côte d'ivoire : Zones importantes pour la pêche : in Nature et faune, volume 24, $\mathrm{n}^{\circ}$ 1, FAO, Rome pp 85-93.

6. Errami E., Ennih N., Bendaoud A., Bouzidi O., Chabou M.C., AndrianaIvo L., Ben Ismail-Lattrache K., Hasina M. (2013) Inventaire 
du géopatrimoine en Afrique : état des lieux et perspectives. in Egoroff G., Cornée A., De Wever P. \& Lalanne A., (eds.) ;- Actes du colloque "Géopatrimoine, un lustre d'inventaire en France », Digne-les-Bains. - Mém. H.S. Soc. géol. France; 10-12 octobre 2012, 13, 128-139.

7. Errami E., Schneider G., Ennih N., Nirina Randrianaly H., Bendaoud A., Noubhani A., Norman N., Allan M., Vasconcelos L., Costa L., AlWosabi M., Al-Subbary A. Mabvuto-ngwira P., Okunlola G., Lawal Halliru S., L. Andrianaivo L., Siby S., Ketchemen B., Gauly M., Hassine M, Azki F., Tea J., Lattrache K., Omulo M. and P. Bobrowsky (2015) Geoheritage and Geoparks in Africa and the Middle-East: Challenges and Perspectives. In Geoheritage to Geoparks: Case Studies from Africa and Beyond. E. Errami, M. Brocx, \& V. Semeniuk (eds), 3-23. Springer Verlag. ISBN; 978 - 3- 319 - 10707 - 3.

8. Gnanzou A. (2014) Etude des séries volcano-sédimentaires de la région de Dabakala (Nord-Est de la Côte d'Ivoire): genèse et évolution magmatique. Contribution à la connaissance de la minéralisation aurifère de Bobosso dans la série de la Haute-Comoé. Thèse Université Paris sud Orsay, France et Université Félix Houphouët Boigny d'Abidjan Côte d'Ivoire. 303 p.

9. Grandgirard V. (1999) L'évaluation des géotopes. Geologia Insubrica 4: pp 59-66

10. Iosif D. (2014) La fiche d'évaluation des géosites. Un exemple exhaustif utilisé sur des sites Roumains. Cinq Continents 4 (10): 158180.

11. Jonin M., Avoine J., Balme C., Gilly Y., Cassagne B., Hango M., Moreno P., Ponet D., Rosenthal P., Fiers V., Buttifant A. (2007) : Patrimoine Géologique. Les réserves naturelles de France. pp.1 - 16

12. Le Loeuff P. \& Marchal E. (1993) Géographie littorale. Le milieu marin. Paris Orstom, pp 15-22 Faculté des lettres et sciences humaines, Université de Lomé (Togo), p 112-125.

13. Lys M. (1961) Études paléontologiques et géologiques de la fal aise de Fresco (Côte d'Ivoire). Ann. Fac. Sci. Univ. Dakar, 6. pp 4780.

14. Martin J-P. (2011) Intégration du patrimoine géologique à la stratégie de développement local du parc naturel du Massif des Bauges (France). Maîtrise en Sciences de l'environnement. Université du Québec à Montréal. 206 p.

15. Martin L. \& Tastet J.P. (1972) Le quaternaire du littoral et du plateau continentale Côte d'Ivoire; rôle des mouvements tectoniques et eustatiques. Ass, Sénégal. Et. Quatern. Ouest afr. Bull. Liaison, Sénégal, n 33-34, pp 17-32. 
16. Mondé S. (1997). Nouvelles approches de la cartographie du plateau continental de la Côte d'Ivoire: aspects morphologiques et sédimentologiques, Thèse Doctorat. Univ. Abidjan- Cocody (Côte d'Ivoire), 58p.

17. Poirier B. (2008) Identification, évaluation et sélection de géosites potentiels le long du sentier national du Québec dans la MRC des Laurentides: une contribution à l'offre écotouristique régionale des municipalités de Labelle et de la conception. Mémoire de maîtrise en Géographie. Université du Québec à Montréal. 202 p.

18. N'Dri G. A. (2008) Le tourisme littoral dans le Sud-Ouest ivoirien. Géographie. Thèse de Doctorat Université de Cocody - Abidjan, 2001 $363 \mathrm{p}$.

19. Remy J.M. (1960) Études paléontologiques et géologiques sur les falaises de Fresco (Côte d’Ivoire). II-Crustacés. Ann. Fac. Sc. Univ. Dakar, 5, pp 55-64.

20. ReynardE.(2004).L'évaluation des géotopes géomorphologiques en $S$ uisse, in Paysages géomorphologiques - Compterendu du séminaire de 3ème cycle, E. Reynard \& J-P. Pralong, Editors. Institut de Géographie: Lausanne. p. 137-149

21. Reynard E. \& Brilha J. (Edts.) (2018) Geoheritage: assessment, protection and management. Elsevier, Amsterdam, $450 \mathrm{p}$

22. Reynard E., Fontana G., Kozlik L., Scapozza C. (2007) A method for assessing scientific and additional values of geomorphosites. Geographica Helvetica 62(3): pp148-158,

23. Reynard E., Perret A., Bussard J., Grangier L., Martin S. (2016) Integrated Approach for the Inventory and Management of Geomorphological Heritage at the Regional Scale. Geoheritage p 4360.

24. Sombo B. C. (2002) Etude de l'évolution structurale et sismostratigraphique du bassin sédimentaire offshore de Côte d'Ivoire, marge passive entaillée d'un canyon. Thèse Doctorat d'Etat, Univ. Abidjan-Cocody (Côte d'Ivoire), 304p.

25. Spengler A. \& Delteil J.B. (1964) Le bassin secondaire-tertiaire de la Côte d'Ivoire. Bassins sédimentaires du littoral africain D. REYRE, Ass. Sew. géol. afr., Paris, pp. 99-113.

26. RGPH (2014) Recensement général de la Population et de l'Habitat. Rapport de l'exécution et présentation des principaux résultats. Institut National de la Statistique. 49 p.

27. Tastet J. P., Martin L. et Aka K. (1993) Géologie et environnement sédimentaire de la marge continentale de Côte d'Ivoire. Le milieu marin. Paris Orstom, pp 23-61. 
28. Tastet J.P. \& Roques R. (1969).Sur quelques minéraux accessoires des falaises paléocènes de Fresco. Ann. Univ. Abidjan, Sciences, 5, pp 157-174.

29. Tessier F.(1952) Contributions à la stratigraphie et à la paléontologie de la partie ouest du Sénégal (Crétacé et Tertiaire). Bulletin de la Direction des Mines de l'AOF, Dakar, 14, (1), p. 1-267.

30. Tessier F. (1960) Etudes paléontologiques et géologiques sur les falaises de Fresco (Côte d'Ivoire). Introduction. Ann. Fac. Sc. Univ. Dakar, 5, pp 35-53.

31. Yao N. J-P. (2012) Caractérisation sédimentologique, minéralogique, géochimique et biostratigraphique des falaises vives de Fresco : Région de Grand-Lahou . Thèse Université Félix Houphouët Boigny (Côte d'Ivoire). 218 p. 